\title{
Correlation of QTc Prolongation \&COPD Severity
}

\author{
Dr G Jegan MD ${ }^{1}$,Dr B Palani Kumar MD², \\ ${ }^{1}$ Assistant Surgeon, Tamilnadu Medical Service \\ ${ }^{2}$ Assistant Professor, Department Of Medicine, Govt Rajaji Hospital, Madurai
}

\begin{abstract}
:
Context: Cigarette smoking is a major public health problem \& it is one of the most important risk factor for Chronic Obstructive Pulmonary Disease (COPD) which is the $4^{\text {th }}$ leading cause of death worldwide. Apart from smoking environmental pollution mainly occupational exposure of toxic gases plays an important role in developing COPD. Hence treating COPD patients based on their severity reduces mortality \& morbidity, COPD severity assessment is done based on GOLD criteria using spirometry, which has its own limitations, hence there is a need for more simple clinical parameter to assess the severity of COPD.

Aim of the study: To assess the correlation of QTC Prolongation in Electrocardiogram \& severity of COPD.

Settings and Design:Prospective observational study

Materials and Methods: This study is conducted among 100 known COPD patients who were admitted at Government Rajaji hospital, Madurai between January 2014 to September 2014, based on GOLD guidelines they were grouped in to mild, moderate \& severe disease, baseline haemoglobin,oxygen saturation, electrocardiogram, echo cardiogram, non contrast CT chest done, \& the correlation of QTc prolongation \& COPD severity is assessed.

Results: In our study, QTc prolongation greater than 450ms observed in FEV1\% less than 50\% and FEVI/FVC ratio less than 0.59. Hypoxia resulted in secondary polycythemia among severe and very severe COPD patients. Chronic hypoxia has resulted in all Arrhythmogenic complication related to COPD.

Conclusions: Incidence of QTC prolongation is more with severe and very severe COPD. Oxygen saturation less than $90 \%$ were reported to have hypoxia, hence the chance of QTC prolongation is High. QTc interval more than 500 millisecond causes arrhythmia,there by causing sudden cardiac death. Hypoxia alters the cardiac repolarization, resulting in increase in the QTc prolongation and various ventricular arrhythmias. Duration of COPD is also a risk factor for developing arrhythmia and sudden cardiac death.
\end{abstract}

Keywords: Corrected QT interval, Chronic Obstructive Pulmonary Disease, GOLD criteria

\section{Introduction}

COPD is defined as a treatable and preventable disease which is characterized by progressive, persistent air flow limitation with enhanced chronic inflammatory response to noxious particles and gases. Comorbidities and exacerbations contribute to the overall severity in individual patients. COPD is otherwise known as "CHRONIC Systemic Inflammatory Syndrome".

COPD is the 4th leading cause of death worldwide. WORLD HEALTH ORGANISATION estimated that 210 million people have moderate to severe chronic obstructive pulmonary disease.COPD is one of the fastest increasing burden to the health care system. The mortality from COPD is expected to increase more than double by 2030.Cigarette smoking itself now regarded as a disease, is major risk factor. It is the most recognized cause among $80 \%$ of the male patients. Smoking, a risk factor to COPD and its comorbidities associated with cardiovascular system. It is an embracing heterogeneous condition with various pathological processes including small airway disease such as chronic bronchiolitis, emphysema and chronic bronchitis. It exhibits multiple systemic components such as weight loss, impaired nutrition and dysfunction of skeletal muscles. The early recognition and treatment plays a vital role in the prognosis of the COPD patients.

Severe and very severe COPD patients are more prone for arterial blood gas abnormalities in the form of hypoxia and respiratory acidosis. Chronic hypoxia manifest in the form of polycythemia. Hypoxia alters the cardiac repolarisation pattern, it ends in the form of prolonged QTc interval in 12 leads electrocardiogram. Prolongation of QTc interval and increased QT dispersion resulting in sudden cardiac death due to ventricular arrhythmias.

\section{Materials And Methods}

Study population:

The study is a Prospective observational Study conducted among 100 COPD patients who are admitted at Government Rajaji hospital, Madurai, from january 2014 to September 2014. 


\section{Inclusion criteria:}

Patients of both gender between the age group of 40-75 years who are diagnosed to have COPD based on GOLD criteria are included in the study

\section{Exclusion criteria:}

Known case of systemic hypertension,Aortic or cardiac disease.history of previous lung disease other than COPD,Medications affecting QT duration,Diabetic patients both Type 1 and Type 2.patients are excluded from the study group

Ethical committee approval: obtained

\section{Study Protocol}

Patients between age of 40-75 years who are already diagnosed to have COPD on regular follow up including both the sex who got admitted in government Rajaji hospital with acute exacerbation for COPD were selected in to the study after screening them for exclusion criteria, informed consent from all patients will be obtained,detailed clinical history \& physical examination will be done in allpatients. investigations like haemoglobin,oxygen saturation using pulse oximetry,ECG,echocardiogram will be done in all,based on Spirometry values applying GOLD guidelines all these patients will be grouped in to mild,moderate,\& severe,corrected QT interval using Bazett's formula will be calculated \& its correlation to COPD severity is assessed

\section{Statistical Analysis}

The information collected regarding all the selected cases were recorded in a master chart. Data analysis was done with the help of computer by using SPSS software and Sigma Stat 3.5 version (2012). Using this software, percentage, mean, standard deviation and ' $p$ ' value were calculated through One way ANOVA, Pearson correlation and Chi square test and $\mathrm{P}$ value of $<0.05$ was taken as significant.

\section{Results}

In the study of 100 patients $40 \%$ of patients belonged to the age group of 61-70 years. And 39\% belonged to 51-60 years. 21\% belonged to 41-50 years. And the mean QTC was $455 \mathrm{~ms}, 446.4 \mathrm{~ms}, 430 \mathrm{~ms}$ respectively,On comparing age and QTC 9 patients in age group of 41-50,18 patients in 51-60 and 19 patients 61-70 belonged to $>450 \mathrm{~ms}$. Out of 100 patients 72 percent belonged to male and 28 percent were females.

The mean of male subjects is $452.92 \mathrm{~ms}$ and female is $400.5 \mathrm{~ms}$. This signifies females have low QTC when compared to males.Among 72 males 39 males and among 28 females 7 females had QTC > 45O ms.In these people $54 \%$ were smokers and 46 percent were non smokers. Among the smokers the mean QTC was 455 $\mathrm{ms}$. And non smokers had $436 \mathrm{~ms}$ which shows significant statistics $\mathrm{p}<0.024$.In this study patients were categorized by FEV1 as severe moderate and mild.26 patients were in severe, 14 patients are in moderate and 60 patients are in mild stages. On comparing with FEV1/FVC the mean was found to be $0.45,0.55,0.65$ respectively.

The mean QTc was $478.46 \mathrm{~ms}, 476.43 \mathrm{~ms}, 422.5 \mathrm{~ms}$ respectively implying higher the FEV1 lesser the QTc interval with good saturation.On comparing SPO2 the study population is divided into 4 groups. In the first group with SPO2 80-85,13 patients were there with mean FEV1 33.84, FEV1/FVC 0.49 mean QTc 501.5 and hemoglobin 17.38.Next group having SPO2 86-90 34 patients were there with mean FEV1 41.76 ,FEV1/FVC 0.54 and mean QTc 479.71 and HB $16.44 \mathrm{mg} / \mathrm{dl}$.In the next group with SPO2 91-95 6 were there with mean FEV1 63.17,FEV1/FVC 0.63 and mean QTC and $\mathrm{Hb} 431.6713 .17 \mathrm{mg} / \mathrm{dl}$ respectively.

In the final group 47 patients had SPO2 >95 and mean FEV1 62.21 FEV1/FVC 0.63 and QTc 408.94 and $\mathrm{Hb} 13.62 \mathrm{mg} / \mathrm{dl}$,In this study 46 patients had $\mathrm{Hb}<15 \mathrm{mg} / \mathrm{dl}$ and the mean QTC was $421.3 \mathrm{~ms}$ and $54 \mathrm{had} \mathrm{Hb}$ $>15$ with mean QTc $467.78 \mathrm{~ms}$ which has statistical significance of $\mathrm{p}<0.001$.

Comparing age VS QTc mean

\begin{tabular}{|l|l|l|l|}
\hline AGE & No.Of cases & MEAN QTc & SD \\
\hline $41-50$ & 21 & 430 & 37.01 \\
\hline $51-60$ & 39 & 446.4 & 40.49 \\
\hline $61-70$ & 40 & 455 & 43.15 \\
\hline
\end{tabular}

Sex vs QTc mean

\begin{tabular}{|l|l|l|l|}
\hline SEX & No.of CASES & QTc Mean & SD \\
\hline MALE & 72 & 452.92 & 40.54 \\
\hline FEMALE & 28 & 429.64 & 40.05 \\
\hline
\end{tabular}




\begin{tabular}{|l|l|l|l|}
\multicolumn{5}{|}{ Mean QTc in smokers } \\
\hline Smokers & No.of cases & QTc mean & SD \\
\hline Yes & 54 & 455 & 41.06 \\
\hline No & 46 & 436.3 & 40.24 \\
\hline
\end{tabular}

Mean QTe vs Hb

\begin{tabular}{|l|l|l|l|}
\hline $\mathrm{Hb}$ & No.of cases & QTc mean & SD \\
\hline$<15$ & 46 & 421.3 & 29.86 \\
\hline$>15$ & 54 & 467.78 & 38.15 \\
\hline
\end{tabular}

Fev1 Vs Spo2

\begin{tabular}{|l|l|}
\hline FEV1 & SPO2 \\
\hline$<30 \%(26)$ & 87.67 \\
\hline $30-49 \%(14)$ & 89.07 \\
\hline $50-79 \%(60)$ & 95.22 \\
\hline
\end{tabular}

Comparison of multiple parameters

\begin{tabular}{|l|l|l|l|l|l|}
\hline SPO2 & No.of cases & FEV1 & FEV1/FVC & QTC & Hb \\
\hline $80-85$ & 13 & 33.84 & 0.49 & 501.54 & 17.38 \\
\hline $86-90$ & 34 & 41.76 & 0.54 & 479.71 & 16.44 \\
\hline $91-95$ & 6 & 63.17 & 0.63 & 431.67 & 13.17 \\
\hline$>95$ & 47 & 62.21 & 0.63 & 408.94 & 13.62 \\
\hline
\end{tabular}

\section{Discussion}

Altered pattern of cardiac repolarization has been denoted as risk factor for ventricular fibrillation and ventricular tachycardia resulting in sudden cardiac death.There is an increased risk of mortality and cardiovascular morbidity in chronic obstructive pulmonary patients. There is a three fold risk of ventricular arrhythmia in COPD atients. Approximately severe COPD and very severe COPD patients had an alteredpattern of repolarization and increased dispersion due to hypoxia. It exposes thesepatients to arrhythmias particularly ventricular fibrillation and ventricular tachycardia resulting in sudden cardiac death. QTc prolongation in a 12 lead ECG denotes summation ventricular depolarization and repolarisation. Its prolongation more than $450 \mathrm{~ms}$ provokes the arrhythmia by inducing reentry tachycardia.

In our study ,out of the 100 patients ,54 patients were found to be smokers whose QTc mean was $455 \mathrm{~ms}$, and in the 46 non smokers QTc mean was $436.3 \mathrm{~ms}$. It confers smokers are more prone for hypoxia than the non smokers. In smokers the incidence of COPD raises with increase in the pack years. Smoking index also plays a major role in contributing the incidence of Pulmonary diseases. The smokers has to be frequently monitored with spirometry values and should be advised to quit smoking. Smoking cessation programmes has to be implemented .14 cases with FEV1 value in the range of 30-49\% indicates severe COPD according to GOLD guidelines. Their QTc mean was $476.43 \mathrm{~ms}$. It signifies that severe COPD patients with chronic history and multiple exacerbation resulted in severe Hypoxic insults and it is manifested in the form of altered cardiac repolarization. 26 cases with FEV1 value in the range of $<30 \%$ indicates very severe COPD according to GOLD guidelines. Their QTc mean was $478.46 \mathrm{~ms}$. This cases has to be monitored with caution since they are more prone for sudden cardiac death due to QTc prolongation.60 cases with FEV1 value in the range of 50-79\% indicates moderate COPD according to GOLD guidelines. Their QTc mean was $425.5 \mathrm{~ms}$. This cases has to be evaluated periodically. Reversal of mortality can be encouraged by smoking cessation. 27 cases with FEV1/FVC value in the range of 0.4-0.49 indicates very severe COPD according to GOLD guidelines. Their QTc mean was $478.52 \mathrm{~ms}$. This cases are in the range of vulnerable re-entry tachycardia, it is a cause for sudden cardiac death among COPD individuals.

47 cases with oxygen saturation less than $90 \%$ had their Hemoglobin value greater than $15 \mathrm{gm} \%$. It signifies that secondary polycythemia among chronic COPD patients. 46 cases had hemoglobin values less than $15 \mathrm{gm} \%$ with QTc mean of $421.3 \mathrm{~ms}$ and their oxygen saturation are greater than $91 \% .54$ cases had Hemoglobin value greater than $15 \mathrm{gm} \%$ with QTc mean of $467.78 \mathrm{~ms}$ and their oxygen saturation are less than $90 \%$.The correlation co-efficient between FEV1\% and FEV1/FVC is 0.92 . It indicates Very high correlation. Reduction in FEV1\% is directly proportional to the FEV1/FVC ratio.The correlation co-efficient between FEV1\% and QTc is -0.597. It indicates Negative moderate correlation. Reduction in FEV1 \% is manifested with increase in the QTc prolongation in the 12 lead Electrocardiogram. The correlation co-efficient between FEV1\% and SPO2 is 0.625. It indicates High correlation. Reduction in the FEV1\% is associated with reduction in the oxygen saturation.

The correlation co-efficient between FEV1/FVC and QTc is -0.556. It indicates Negative moderate correlation. Increase in the FEV1/FVC is manifested with decrease in the QTc prolongation in the 12 lead electrocardiogram. The correlation co-efficient between QTc and SPO2 is -0.944. It indicated Negative very high correlation. Increase in the QTc prolongation is associated with decrease in the oxygen saturation.The 
correlation co-efficient between QTc and $\mathrm{Hb}$ is 0.605. It indicates Highcorrelation. Increase in the QTc prolongation is associated with Increase in the Hb.In our study, QTc prolongation was observed among individuals with severe and very severe COPD.

\section{Conclusion}

Incidence of hypoxia and QTc prolongation were directly proportional among severe and very severe COPD patients. Hypoxia alters the cardiac repolarization resulting in the prolongation of ventricular activation time, and it should be monitored periodically. Domestic oxygen delivery system should be implemented to these individuals to reduce the cardio vascular morbidity and mortality. Home oxygen delivery system plays a major role in reversing and reducing the progression of COPD. Smoking cessation plays a vital role in

reducing the mortality of COPD. So in our study, QTc prolongation greater than 450ms observed in FEV $1 \%$ less than 50\% and FEV1/FVC ratio less than 0.59. Hypoxia resulted in secondary polycythemia among severe and very severe COPD patients. Chronic hypoxia has resulted in all Arrhythmogenic complication related to COPD. Incidence of QTc prolongation is more with severe and very severe COPD. Oxygen saturation less than $90 \%$ were reported to have hypoxia, hence the chance of QTc prolongation is High. QTc interval more than 500 millisecond causes arrhythmia,there by causing sudden cardiac death. Hypoxia alters the cardiac repolarization, resulting in increase in the QTc prolongation and various ventricular arrhythmias. Duration of COPD is also a risk factor for developing arrhythmia and sudden cardiac death.

\section{References}

[1]. Anthonisen NR, Connett JE, Enright PL, Manfreba J: Hospitalizations andmortality in the lung health study. AM J Respir crit care Med 2002, 166:333-339.

[2]. Mcgarvey LP, John M, Andreson JA, Zvarich M, wise RA:Ascertainment of cause- specific mortality in COPD:Operations of the TORCH clinical end pointcommity. Thorax 2007, 62:411-415.

[3]. Claren Bach CF, Senno, Sievi NA, Cammen G, Van Gestel AJ, Rossi VA,Puhan MA, Thurnheer R, Russi EW, Kohier M: determinants of endothelial.

[4]. Sin DD, Man SF: Chronic obstructive pulmonary disease as a risk factor forcardio vascular morbidity and mortality. Proc AM Thorac SOC 2005, 2:8-11.

[5]. Elming H, Bren Dorp B, Koper L, Sahebzadah N, Torp-petersen C, QTc intervalin the assessment of cardiac case.Chord electro physical rev 2002, 6:289-294.

[6]. Bednar MM, Harrigan EP, Anziano RJ, Camm AJ, Ruskin JM, the QT interval.Prog cardio vascular disease 2001, 43:1-45.

[7]. Antzelevitch C, Shimizu W, Yan GX, Sicouri S, cellular basics for QTdispersion. J electro cardio 1998, 80(suppl):168-175

[8]. Barr CS, Naas A, Freeman M, Lan CC, Struthers AD:QT dispersion and suddenunexpected death in chronic heart failure.Lancet 1994, 343:327-329.

[9]. Yamaguchi M, Shimizu M, Ino H, Terai H, Uchiyama K, Oe K, Mabuchi T,Konno T, Kaneda T, Mabuchi H:T wave peak to end interval and QT dispersion inacquired in long QT syndrome. A new index for arrhythmogenicity. Clin SD(lond)2003, 105:671-676.

[10]. Robins J, Nelson JC, Rautaharju PM, Gottdiener JS, the association betweenthe length of the QT interval and mortality in the cardio vascular health study.AMJ med 2003, 115:689-694.

[11]. Schuneman HJ, Dorn J, Grant VJ, Winkelstein W Jr, Trevisan M, pulmonaryfunction is a long term predictor of mortality in a general population. 29 yearfollow up of the buffalo health study. Chest 2000, 118:656-664.

[12]. Buch P, Friberg J, Scharling H, Lange P, Prescott E, Reduced lung functionand risk of atrial fibrillation in the Copenhagen city heart study. Eur respire J 2003,21:1012-1016.

[13]. Kleiger RE, senior RM, long term electro cardiographic monitoring ofambulatory patients with chronic airway obstruction. Chest 1974, 65:483-487.

[14]. Yildiz P, Tukek T, Akaya V, Sozen AB, Yildiz A, Korkut F, Yilmaz B,Ventricular arrhythmias in patients with COPD are associated with QT depression.Chest2002, 122:2055-2061.

[15]. Drugs that prolong QTc interval and /or induce Torsade de pointes.Http://www.atforum.com/siteroute/pages/addiction resources/QT drugs:209-03-

[16]. 02.Pdf.

[17]. Rossi VA, Stoewhas AEC, Caman G, Steffel J, Bloch KE, Stradling Jr, KohlerM, the effects of continuous positive airway pressure therapy withdrawl on cardiacrepolarization:data from a randomized controlled trial. Eurheart J, 2012,33:2206-2212.

[18]. Pickharn D, Hasanien AA, measurement and rate correction of QT interval.AACN adb crit care.2013, 24:90-96.

[19]. Day CP, Mc Comb JM, Campel RW:Qt dispersion: indication of arrhythmia of risk in patients with long QT intervals. BR heart J, 1990, 63:342-344.

[20]. Bazett HC, The time relation of blood pressure changes after excision ofadrenal gland, with some observations on blood volume changes. J Physiol 1920,53:320-339.

[21]. DE bruyne MC, Hoes AW, Kors JA, Hofman A, Vanbemmel JH, GrobbeeDE:Prolonged QT interval predicts cardiac and all causes of mortality in theelderly. The Rotterdam study. EUR heart J, 1999, 20:278-284

[22]. Zipes DP, Wellens HJ, sudden cardiac death.circulation 1998, 98:2334-2351.

[23]. Viskin S: long QT syndromes and torsade de pointes. Lancer 1999, 354:1625-1633. 